\title{
Orientação profissional em atividade física e alimentação: um estudo de caso
}

\author{
Professional guidance on physical activity and nutrition: a case study
}

\author{
Thaynara Nathalie Carvalho Estevam ${ }^{1}$ \\ Mayla de Paula Torres Simplício ${ }^{1}$ \\ Guilherme Pereira Oliveira ${ }^{1}$ \\ Michele Aparecida Almeida ${ }^{1}$ \\ Guilherme de Azambuja Pussieldi ${ }^{1}$ \\ Afonso Timão Simplício ${ }^{1}$ \\ Valéria Cristina de Faria ${ }^{1, *}$
}

\section{Resumo}

Objetivo: Verificar os benefícios da orientação conjunta da educação física e nutrição sobre o condicionamento cardiovascular, parâmetros antropométricos e bioquímicos após quatro meses de intervenção. Métodos: Foi selecionado um participante do Espaço Movimento, do sexo feminino, com 60 anos de idade e hipertensa. A intervenção foi realizada por quatro meses, a atividade física prescrita foi caminhada com a orientação e acompanhamento profissional, com uma duração média de 45 minutos e frequência de três vezes por semana, totalizando 49 treinos no período. A participante contou com a orientação e acompanhamento nutricional individual durante todo o período de intervenção, contando com avaliação inicial e retorno periódico para adequações da dieta. Foram avaliados no início e quatro meses após: frequência cárdica (FC) e pressão arterial (PA) em repouso, índice de massa corporal (IMC), índice cintura/quadril (ICQ), percentual de gordura corporal (\%GC), percentual de massa muscular (\%MM), VO2máx e exames bioquímicos. Resultados: A participante apresentou as seguintes mudanças: aumento da PA de repouso, do \%MM e do VO2máx; redução da FC de repouso, do IMC, do ICQ, do \%GC, e em todos os exames bioquímicos. Conclusão: Diante dos resultados podemos afirmar que uma ação conjunta, exercício físico e alimentação, somado a disciplina e regularidade do participante, são capazes de afetar positivamente os índices de saúde avaliados.

Palavras chave: saúde, atividade física, estudo de caso.

\begin{abstract}
Objective: To assess the benefits of joint guidance of physical education and nutrition on cardiovascular fitness, anthropometric and biochemical parameters after four months of intervention. Methods: We selected a participant of the Espaço Movimento, female, with 60 years of age and hypertensive. The intervention was performed for four months, the prescribed physical activity was walking with professional guidance and monitoring, with an average duration of 45 minutes and frequency of three times a week, totaling 49 training sessions in the period. The participant had the guidance and individual nutritional monitoring throughout the intervention period, with initial assessment and periodic return for diet adjustments. It were assessed at baseline and after four months: heart rate (HR) and blood pressure $(\mathrm{BP})$ at rest, body mass index (BMI), waist / hip ratio (WHR), body fat percentage (\%BF), muscle mass percentage (\%MM), VO2máx and biochemical tests. Results: The participant showed the following changes: increased resting BP, \%MM and VO2max; reduction of resting $\mathrm{HR}, \mathrm{BMI}, \mathrm{WHR}, \% \mathrm{BF}$, and all biochemical tests. Conclusion: Based on the results we can say that a joint action, physical exercise and diet, coupled with disciplined and regular participant are able to positively affect health outcomes evaluated.
\end{abstract}

Keywords: health, physical activity, case study.
Afiliação dos autores

${ }^{1}$ Universidade Federal de Viçosa, Campus Florestal, Florestal, Minas Gerais, Brasil.

${ }^{*}$ Autor correspondente

Rua José de Souza Maciel, 192. Bairro Dona Suzana, CEP: 35.690 000, Florestal, Minas Gerais, Brasil. e-mail: valeriaefiufv@yahoo.com.br

Conflito de interesses

Os autores declararam não haver conflito de interesses.

Processo de arbitragem

Recebido: 15/12/2016 Aprovado: 25/04/2017 


\section{Introdução}

A prática de atividade física associada à dieta traz inúmeros benefícios na prevenção e combate de doenças crônicas não transmissíveis, uma vez que prescritas adequadamente ${ }^{1}, 2$. Nessa perspectiva, é válido ressaltar que os benefícios são ainda maiores para os idosos, considerando o processo natural de envelhecimento e seus deletérios ${ }^{2},{ }^{3}$.

Dentro desta ótica, é comprovado cientificamente que, apesar de não reverter esse quadro, é possível desacelerá-lo, através de uma intervenção eficaz no estilo de vida do indivíduo ${ }^{2-4}$. A prescrição de exercícios deve considerar resultados de uma avaliação prévia a intervenção e buscar contemplar os componentes da aptidão física, a individualidade biológica do sujeito, e as variáveis duração, frequência, intensidade e modalidade ${ }^{4}$.

Pesquisadores ${ }^{2,4,5}$ afirmam que a intensidade da atividade física para o idoso não precisa ser intensa para gerar benefícios à saúde, tais como, melhora da capacidade cardiorrespiratória, nível de força muscular, manutenção ou melhora da densidade mineral óssea, melhora da qualidade de vida, entre outros, além dos benefícios psicológicos, como melhora da autoestima e autoconfiança.

Estudos $^{6-8}$ realizados com o público idoso demonstram a importância da informação a cerca da associação da atividade física e a alimentação para a mudança do estilo de vida, e os benefícios adquiridos.

Visto que a atividade física regular propicia uma melhora na expectativa e qualidade de vida do indivíduo idoso, e que associada à alimentação regular e balanceada os resultados podem ser potencializados, o objetivo desse estudo foi verificar os benefícios da orientação conjunta da educação física e nutrição sobre o condicionamento cardiovascular, parâmetros antropométricos e bioquímicos após quatro meses de intervenção.

\section{Métodos}

\section{Desenho do estudo}

Trata-se de um estudo de caso do tipo avaliativo, a partir do qual se pretende avaliar o mérito da modalidade grupo de caminhada/corrida orientada recentemente implantada no Espaço Movimento em conjunto com orientação nutricional.

\section{Espaço Movimento (Serviço Agros de Atividade Física e Saúde)}

O Serviço Agros de Atividade Física e Saúde é uma parceria do Agros - Instituto UFV de Seguridade Social (Agros), empresa de plano de saúde e previdência que se destina a atender os servidores da Universidade Federal de Viçosa - Campus Florestal (UFV-CAF) e seus dependentes, com a própria UFVCAF.

O Serviço tem como objetivo geral promover saúde e prevenir contra doenças não transmissíveis, e no intuito de atingir um público de diversas idades são desenvolvidas atividades regulares e eventos periódicos que abordem essa temática. Uma das atividades regulares oferecidas é o Grupo de Caminhada/Corrida Orientada (GCO), do qual foi selecionado um participante para esse estudo de caso.

\section{Participante do estudo}

Foi selecionado para o estudo um participante do GCO do sexo feminino, com 60 anos de idade, com diagnóstico de hipertensão arterial sistêmica e dislipidemia.

\section{Cuidados éticos}

Antes de iniciar a intervenção a participante obteve informacões e esclarecimentos sobre o estudo, e assinou o termo de consentimento livre e esclarecido. Considerando o cunho de pesquisa a proposta do Espaço Movimento foi apreciada e aprovada pelo Comitê de Ética em Pesquisa com Seres Humanos da Universidade Federal de Viçosa, conforme determina a Resolução no 466/12 do Conselho Nacional de Saúde, sob o parecer número 487.635.

\section{Intervenção}

Inicialmente a participante foi submetida a uma avaliação física, na qual foram mensuradas variáveis hemodinâmicas em repouso, frequência cardíaca (FC) e pressão arterial (PA); dados antropométricos, massa corporal e estatura, para o cálculo do índice de massa corporal (IMC); circunferências de cintura e quadril, para o cálculo do índice cintura-quadril; e os percentuais de gordura corporal (\%GC) e massa magra (\%MM) (100 - \%GC) através do protocolo de Faulkner ${ }^{9}$.

$\mathrm{Na}$ sequência a participante foi avaliada clinicamente por um médico frente à realização de teste ergométrico e exames bioquímicos (glicemia de jejum, colesterol total e frações, triglicérides e ácido úrico).

Após a liberação médica foi realizado um teste de campo para avaliação do $\mathrm{VO}_{2}$ máx ${ }^{10}$, e iniciou-se a prescrição da atividade física, que consistia da participação do GCO com frequência semanal de três vezes e duração média de 45 minutos por sessão de treino, com intensidade leve a moderada, a qual foi controlada pela escala de percepção de esforço (escala de Borg) ${ }^{11}$.

Concomitante a prática física a participante contou com acompanhamento nutricional individual, o qual consistiu de uma avaliação inicial para prescrição da dieta e avaliações periódicas para a adequação desta.

A intervenção durou quatro meses, totalizando 49 treinos realizados, e ao final foram repetidos as mensurações da avaliação física e os exames bioquímicos. A figura 1 apresenta 0 fluxograma com as etapas de intervenção.

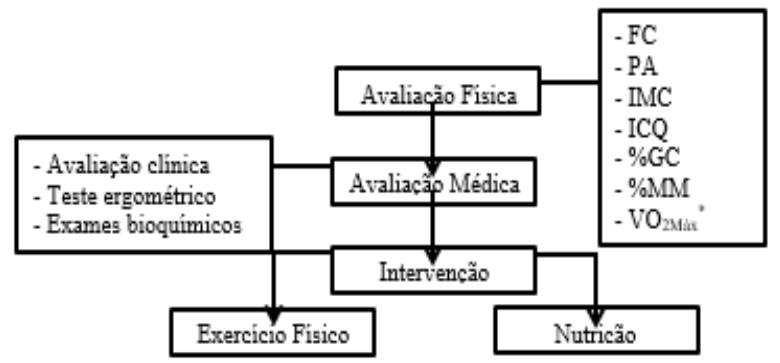

Figura 1. Fluxograma das etapas de intervenção. *Avaliação feita após a liberação médica como última etapa da avaliação física.

\section{Resultados}

$\mathrm{Na}$ tabela 1 são apresentados os valores obtidos na avaliação física e médica antes da intervenção e após a intervenção. Com exceção da PA e HDL todas as medidas sofreram alterações positivas após a intervenção.

Tabela 1

Resultados dos índices de saúde avaliados no participante voluntário da pesquisa.

\begin{tabular}{lcc}
\hline \multicolumn{1}{c}{ Índices de saúde } & Pré intervenção & Pós intervenção \\
\hline FC repouso (bpm) & 69 & 58 \\
PA repouso (mmHg) & $140 / 100$ & $170 / 95$ \\
VO2Máx (ml.kg.min) & 23,80 & 26,16 \\
IMC $\left(\mathrm{kg} / \mathrm{m}^{2}\right)$ & 28,45 & 26,29 \\
ICQ & 0,81 & 0,79 \\
$\%$ GC & 26,21 & 20,97 \\
$\% M M$ & 73,79 & 79,03 \\
Glicemia $(\mathrm{mg} / \mathrm{dL})$ & 100 & 99 \\
CT $(\mathrm{mg} / \mathrm{dL})$ & 242 & 147 \\
HDL $(\mathrm{mg} / \mathrm{dL})$ & 69 & 64 \\
LDL $(\mathrm{mg} / \mathrm{dL})$ & 152 & 72 \\
Triglicérides $(\mathrm{mg} / \mathrm{dL})$ & 107 & 56 \\
Ácido úrico $(\mathrm{mg} / \mathrm{dL})$ & 5,8 & 5,5 \\
\hline
\end{tabular}

\section{Discussão}

Os benefícios advindos das duas intervenções são esperados desde que estas sejam prescritas adequadamente, e ficam mais claros quando há compromisso e disciplina do indivíduo atendido, o que ocorreu no nosso estudo.

As mudanças observadas no comportamento cardiovascular, $\mathrm{FC}$ de repouso e $\mathrm{VO}_{2}$ máx, são devidas às adaptações crônicas ao treinamento aeróbico, pois se sabe que esse perfil de exercício aumenta a atividade parassimpática e atenua a atividade simpática acarretando em uma bradicardia de 
repouso, e como uma adaptação mais significativa ocorre um aumento do débito cardíaco máximo, devido principalmente por um volume sistólico aprimorado com o treinamento, atingindo assim níveis mais altos de VO2máx3.

Em contrapartida a PA apresentou uma mudança negativa, o que pode ser devido à influência de fatores emocionais ${ }^{12}$, já que esta participante é demasiadamente envolvida e responsável em diversas tarefas no âmbito familiar e se encontra frequentemente sob situações de estresse.

Segundo as Diretrizes Brasileiras de Obesidade ${ }^{13}$ para o idoso é aceitável um IMC entre 22 e 27, portanto nossa participante migrou de sobrepeso para o peso adequado após intervenção, e esse comportamento ocorreu devido a uma melhora de composição corporal, redução do \%GC e aumento do \%MM, reduzindo algumas das alterações advindas do envelhecimento ${ }^{2,3}$.

Quanto aos resultados dos exames bioquímicos o que mais se destaca é a mudança do perfil lipídico, principalmente na redução do colesterol total, LDL e triglicérides, enquanto que o $\mathrm{HDL}$ ao contrário do que se esperava apresentou uma pequena redução, mas que ainda assim se manteve de acordo com o recomendado ${ }^{14}$. Essas alterações reforçam o posicionamento de Fagherazzi et al. ${ }^{15}$ de que a educação nutricional continuada associada à atividade física regular é importante para a efetividade do controle das dislipidemias.

A nova proposta de modalidade regular do Espaço Movimento, GCO, parece ser efetiva em contribuir com a ação nutricional no que diz respeito às variáveis cardiovasculares, antropométricas e lipídicas, favorecendo dessa forma um envelhecimento saudável e a prevenção de eventos cardiovasculares.

\section{Conclusão}

Diante dos resultados podemos afirmar que uma ação conjunta, exercício físico e alimentação, somado a disciplinada e regularidade do participante, são capazes de afetar positivamente os índices de saúde avaliados.

\section{Referências}

1. Coelho C, Burine RC. Atividade física para prevenção e tratamento das doenças crônicas não transmissíveis e da incapacidade funcional. Rev Nutr 2009; 22(6): 937-946.

2. 2- Nahas MV. Atividade física, saúde e qualidade de vida: conceitos e

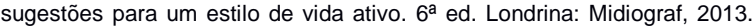
$335 \mathrm{p}$.

3. 3- Mcardle WD, Katch FI, Katch VL. Fisiologia do Exercício: Energia, Nutrição e Desempenho Humano. 5. ed. Rio de Janeiro: Ganabara Koogan S.a, 2003. 1113 p.

4. Nobrega ACL, et al. Posicionamento oficial da Sociedade Brasileira de Medicina do Esporte e da Sociedade Brasileira de Geriatria e Gerontologia: atividade física e saúde no idoso. Rev Bras Med Esporte 1999; 5(6): 207-211.

5. Matsudo SM, Matsudo VKR, Barros Neto TL. Atividade física e envelhecimento: aspectos epidemiológicos. Rev Bras Med Esporte 2001; $7(1): 2-13$.

6. Ferreira $\mathrm{M}$, et al . Efeitos de um programa de orientação de atividade física e nutricional sobre o nível de atividade física de mulheres fisicamente ativas de 50 a 72 anos de idade. Rev Bras Med Esporte 2005; 11(3): 172 176.

7. Cavalcanti $\mathrm{CL}$, et al . Programa de intervenção nutricional associado à atividade física: discurso de idosas obesas. Ciênc saúde coletiva 2011: 16(5): 2383-2390.

8. Burke L, et al. Physical activity and nutrition behavioural outcomes of a home-based intervention program for seniors: a randomized controlled trial. Int J Behav Nutr Phys Act 2013; 10(1): 1-8.

9. Faulkner JA. Physiolgy of swimming and diving. In: H. FALLS. Exercise Physiology, Baltimore: Academic Press, 1968.

10. Cooper $\mathrm{KH}$. A means of assessing maximal oxygen intake: correlation between field and treadmill testing. JAMA 1968; 203: 135-138.

11. Borg GAV. Psychophysical basis of perceived exertion. Med Sci Sports Exerc 1982; 14: 377-381.

12. Fonseca FCA, Coelho RZC, Nicolato R, Malloy-Diniz LF, Filho HCS. A influência de fatores emocionais sobre a hipertensão arterial. J Bras Psiquiatr 2009; 58(2):128-134.

13. Associação Brasileira para o Estudo da Obesidade e da Síndrome Metabólica. Diretrizes brasileiras de obesidade 2016 / ABESO Associação Brasileira para o Estudo da Obesidade e da Síndrome Metabólica. - 4.ed. - São Paulo, SP.

14. Sociedade Brasileira de Cardiologia. V Diretriz Brasileira de Dislipidemias e Prevenção da Aterosclerose. Arq Bras Cardiol 2013; 101(4Supl.1): 1-22.

15. Fagherazzi S, Dias RL, Bortolo F. Impacto do Exercício Físico Isolado e Combinado com Dieta Sobre os Níveis Séricos de Hdl, Ldl, Colesterol Total e Triglicerídeos. Rev Bras Med Esporte 2008; 14(4): 381-386. 